\title{
3.2 Die lufthygienischen Zusammenhänge
}

\subsubsection{Einleitung}

Emission

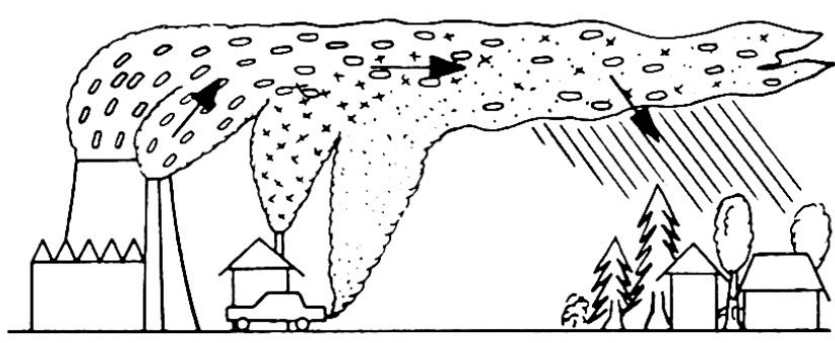

Der gesamte Problemkomplex der Lufthygiene gliedert sich in drei Teilbereiche auf:

Emission: Schadstoffquellen oder der Ort, wo die Luftfremdstoffe in die Atmosphäre austreten.

Transmission: Transport, Verfrachtung und Ausbreitung der Luftfremdstoffe in der Atmosphäre.

Immission: Einwirkungsbereich oder die Gebiete, wo die Luftfremdstoffe zur Erdoberfläche zurückgelangen.

\subsubsection{Emissionen}

Luftverunreinigungen sind Veränderungen des natürlichen Zustandes der Luft, namentlich durch Rauch, Ruß, Staub, Gase, Aerosole, Dämpfe, Geruch oder Wärme. Wir unterscheiden grundsätzlich drei Hauptemissionsquellengruppen:

\subsubsection{Verkehrsemissionen}

Im Bereich der Lufthygiene wird der Verkehr als Linienquelle bezeichnet. In weiten Gebieten unseres Landes sind die Verkehrsemissionen das vordringlichste Lufthygieneproblem. Dabei werden folgende Luftfremdstoffe emittiert:

Stickoxide $\left(\mathrm{NO}_{\mathrm{x}}\right)$

Kohlenwasserstoffe $(\mathrm{CH})$

Kohlenmonoxid (CO)

Staub (Schwermetalle, z. B. Blei)

Bereits heute werden in verschiedenen Schweizer Städten die Immissionsgrenzwerte an stark befahrenen Straßen massiv überschritten. In Anbetracht dieser Situation ist eine rasche Verschärfung der Abgasgrenzwerte für Automobile unumgänglich. Dies umso mehr, weil diese Maßnahme erst mit einer gewissen Verzögerungszeit voll wirksam wird. Neben dieser Quellenbekämpfung gibt es auch sekundäre Maßnahmen gegen die Verkehrsabgase, z. B. Straßenführung, Verkehrsflüsse und -umlenkungen usw.

\subsubsection{Hausbrand}

Darunter werden in der Lufthygiene alle Feuerungsanlagen (Öl, Kohle und Holz) verstanden. Viele kleine Feuerungsanlagen (z.B. Quartier) ergeben praktisch eine Flächenquelle. Bei der Verbrennung von fossilen Brennstoffen entstehen hauptsächlich Staub- (Russ-) und Schwefeldioxidemissionen. Zur Herabsetzung dieser Emissionen haben viele Gemeinden und verschiedene Kantone die lufthygienische Ölfeuerungskontrolle eingeführt.

Besondere Probleme im Bereich der Luftreinhaltung bieten Feuerungsanlagen mit weniger sauberen Brennstoffen, wie beispielsweise

Schwerölfeuerungen

Kohlefeuerungen

Altölfeuerungen

Kehricht- und Sonderabfallverbrennung

Hierbei können äußerst schädliche Emissionen entstehen (z. B. Schwermetalle, Kohlenwasserstoffe, Fluor usw.) und in die Atmosphäre austreten. So ist es heute nicht mehr denkbar, daß beispielsweise eine Kehrichtverbrennungsanlage ohne Rauchgasreinigung betrieben werden kann.

\subsubsection{Industrie, Gewerbe und andere Betriebe}

Das Spektrum der hier emittierten Luftfremdstoffe ist sehr breit und reicht vom relativ harmlosen Wasserdampf bis zu schädlichen Luftverunreinigungen. Daneben gibt es auch Emissionen, die nur belästigend sind (z.B. Gerüche). Zwei Aspekte sind für diese Punktquellen wichtig: Einerseits ist die Konzentration der an die Atmosphäre abgegebenen Luftfremdstoffe wichtig (z. B. $\mathrm{mg} / \mathrm{m}^{3}$ oder $\mathrm{ppm}$ ), und anderseits ist es der Massenstrom der Luftfremdstoffe (z. B. kg/h oder ta).

Hans Mathys, Dr., Abteilungsleiter Lufthygiene Abteilung Lufthygiene des Kantonalen Amtes für Industrie, Gewerbe und Arbeit, Laupenstrasse 2, 3008 Bern 


\subsubsection{Zusammenfassung}

Luftfremdstoffe treten in gasförmiger, fester, flüssiger Form oder in Kombination (Aerosole) auf. Sie werden je nach ihrer Schädlichkeit in drei Klassen aufgeteilt, und dementsprechend werden auch die Emissionsgrenzwerte festgelegt. Somit kann die oftmals gestellte Frage nach der lufthygienischen Gesamtbelastung gar nicht abschließend beantwortet werden. Fest steht aber, daß Lufthygieneprobleme nur emissionsseitig, also an der Quelle, gelöst werden können (z. B. durch technische abluftseitige Maßnahmen, Änderungen in der Prozeßführung usw.). Deshalb sind die Emissionsgrenzwerte für den praktischen Bereich der Luftreinhaltung von zentraler Bedeutung.

\subsubsection{Transmission}

Nach ihrem Eintritt in die Atmosphäre werden die Luftfremdstoffe je nach den äußern Verhältnissen verfrachtet und ausgebreitet. Während des Transportes können sie sich sowohl in ihrer chemischen Zusammensetzung als auch physikalisch verändern. Dabei treten auch synergistische Effekte auf (Zusammenwirken mehrerer Luftfremdstoffe). Für die Ausbreitung und den Transport der Luftschadstoffe sind folgende Elemente von Bedeutung:

\subsubsection{Topographie und Bodenbeschaffenheit}

Geländeformen wie Bergketten, Hügel, Schluchten, Täler, Engnisse usw. wirken sich stark auf die Ausbreitung der Luftfremdstoffe aus. Es entstehen Ablenkungs-, Kanalisations-, Beschleunigungs-, Abschirmund Turbulenzeffekte. Aber auch die Bodenbeschaffenheit (= Bodenrauhigkeit) kann die Ausbreitungsmechanismen nachhaltig beeinflussen, z. B. Hecken, Wälder, Baukörper, Asphaltfläche usw.

\subsubsection{Windverhältnisse}

Entscheidend für die Verdünnung und Ausbreitung von Schadstoffen sind die Windgeschwindigkeit und Windrichtung (= Windfeld). Die großräumigen Starkwinde (Strömungsfeld zwischen den Druckzentren), wie wir sie etwa in Norddeutschland oder Dänemark antreffen, werden durch unser intensives Relief im Mittel bis zu $60 \%$ reduziert. Erst in großer Höhe über der Erdoberfläche treffen wir ungestörte Windverhältnisse an. In unserem Land spielen deshalb oft regionale oder lokale Windsysteme für die Ausbreitung von Luftfremdstoffen eine übergeordnete Rolle, z. B. See-/Landwinde, Berg-/Talwinde.

\subsubsection{Inversionen}

Bei der Ausbreitung der Luftfremdstoffe spielen auch die vertikalen Luftbewegungen (Turbulenz) eine wichtige Rolle. Dabei gibt es einen Sonderfall, den es besonders zu beachten gilt: die Inversionen. Durch die nächtliche Ausstrahlung entstehen in Mulden, Tälern und Tieflagen sehr oft Kaltluftseen. Die Obergrenze dieser Kaltluftmassen wirkt sich wie eine Sperrschicht aus, so daß vertikale Luftbewegungen kaum mehr möglich sind. Dauern solche Inversionen längere Zeit an, werden Luftfremdstoffe im Kaltluftsee akkumuliert; d.h. der Schadstoffpegel kann bedenklich ansteigen. Inversionen können lokal, kleinräumig, aber auch großräumig (z.B. ganzes Mittelland, Nebelmeer) auftreten.

\subsubsection{Zusammenfassung}

Die Ausbreitungsmechanismen der Luftfremdstoffe wurden in der Vergangenheit sehr oft vernachlässigt. Gerade in der Praxis der Luftreinhaltung konzentrierte man sich allzu einseitig auf das Emissionsgeschehen. Obwohl die Transmission für den Menschen nicht beeinflußbar ist, darf deren Rolle nicht unterschätzt werden.

Es ist heute bekannt, daß die Verfrachtung von Luftfremdstoffen oft Hunderte von Kilometern beträgt. Als Beispiel dafür mögen die "sauren Niederschläge» dienen. Solchen großräumigen grenzüberschreitenden Verfrachtungen kann sich auch die Schweiz nicht entziehen; sie wird dadurch je nach der Situation zum Importeur oder Exporteur von Luftverunreinigungen.

Die Ausbreitung von Luftfremdstoffen, so komplex sie auch ist, kann heute berechnet werden. Dazu werden physikalische oder mathematische Modelle verwendet. Solche Verfahren finden ihre Anwendung nicht nur im Forschungsbereich, sondern auch in der Praxis der Luftreinhaltung (z.B. Berechnung von Kaminhöhen).

\subsubsection{Immissionen}

Die Immissionen sind das eigentliche Produkt aus Emission und Transmission. Wir sind heute aber noch weit davon entfernt, alle die Prozesse, die sich in den untersten Schichten der Atmosphäre abspielen, lükkenlos zu verstehen. Einerseits wirken die Luftfremdstoffe auf die Biosphäre (Menschen, Tiere und Pflanzen), anderseits wird auch die tote Materie davon betroffen (Bauwerke, Boden und Wasser). Zur Verhütung von Schäden und Störungen sowie zum Gesundheitsschutz des Menschen werden Immissionsgrenzwerte festgelegt. Dabei wird zwischen der Kurz- und Langzeiteinwirkung unterschieden. Es hat sich in neuester Zeit mit aller Deutlichkeit gezeigt (Waldsterben), daß gewisse Pflanzen sehr subtil auf die Luftverschmutzung reagieren und geradezu als Maßstab für den Grad der Luftverunreinigung verwendet werden können. Deshalb darf die Forderung, daß für das Festlegen von Immissionsgrenzwerten nicht nur die menschliche Gesundheit als alleiniger Maßstab dienen kann, nicht mehr unberücksichtigt bleiben. 